\title{
Nonlinear Limits for Single-Crystal Silicon Microresonators
}

\author{
Ville Kaajakari, Tomi Mattila, Member, IEEE, Aarne Oja, and Heikki Seppä
}

\begin{abstract}
Nonlinear effects in single-crystal silicon microresonators are analyzed with the focus on mechanical nonlinearities. The bulk acoustic wave (BAW) resonators are shown to have orders-of-magnitude higher energy storage capability than flexural beam resonators. The bifurcation point for the silicon BAW resonators is measured and the maximum vibration amplitude is shown to approach the intrinsic material limit. The importance of nonlinearities in setting the limit for vibration energy storage is demonstrated in oscillator applications. The phase noise calculated for silicon microresonator-based oscillators is compared to the conventional macroscopic quartz-based oscillators, and it is shown that the higher energy density attainable with the silicon resonators can partially compensate for the small microresonator size. Scaling law for microresonator phase noise is developed.

[1246]
\end{abstract}

Index Terms-Bifurcation, bulk acoustic wave (BAW) devices, hysteresis, microresonators, nonlinear oscillators, nonlinearities, oscillator noise, oscillators, phase noise, resonators.

\section{INTRODUCTION}

A $\mathrm{S}$ the wireless communication devices are becoming ubiquitous, there is a growing need to miniaturize the size-consuming analog RF components. Although the new transceiver architectures such as direct conversion cut down the number of analog filters, a high spectral purity local oscillator is still required. The problem is perhaps the most obvious in the relatively low cost applications such as Bluetooth where the entire communication circuitry, with the exception of the frequency reference and a few capacitors, has been integrated on a single CMOS chip.

Micromechanical silicon resonators are an interesting alternative to the macroscopic quartz resonators due to their compact size and feasibility for integration with IC technologies [1]. Unfortunately, the smaller size of the micromechanical resonators unavoidably results in a lower energy storage and power handling capacity. As a direct consequence, achieving a sufficient phase noise performance becomes a challenge [2]. The maximum power handling capacity is also a critical parameter in filter applications. The central aspect of this paper is, therefore, to provide detailed knowledge of the fundamental nonlinearity mechanisms in microresonators and of the induced energy storage limits. The performance limits are demonstrated in

Manuscript received January 5, 2004; revised March 22, 2004. This work was supported by the Nokia Research Center, Okmetic, STMicroelectronics, VTI Technologies, and the Finnish National Technology Agency. Subject Editor C. Hierold.

The authors are with VTT Information Technology, FIN 02044 VTT, Finland (e-mail: ville.kaajakari@vtt.fi).

Digital Object Identifier 10.1109/JMEMS.2004.835771 oscillator applications and microresonator performance is compared to macroscopic quartz.

The paper is organized as follows: First, the theory of nonlinear oscillations is reviewed in Section II. Expressions to estimate the maximum vibration amplitude (the bifurcation limit) are given and a scaling law for the maximum energy stored in the resonator is derived. In Section III, the various nonlinear effects in electrostatically actuated microresonators are identified. The maximum energy storable in silicon flexural (bridge and cantilever) resonators and bulk acoustic wave (BAW) resonators is compared. It is shown that at the nonlinear limit, the BAW resonators can store orders-of-magnitude more energy than the flexural resonators. In Section IV, the nonlinear analysis of BAW resonators is refined to include material effects. The distributed material nonlinearity is theoretically estimated using the nonlinear engineering Young's modulus. A model incorporating the material effects is developed and simulated with the method of harmonic balance. The simulations are compared to experimental data and it is shown that the energy stored in the BAW resonators approaches the material nonlinearity limit. In Section $\mathrm{V}$, the oscillator phase noise is considered. The equation for phase noise is derived to explicitly show the relation between the stored energy and phase noise. The theoretical phase noise attainable with flexural and BAW resonators is compared to the macro quartz crystal based oscillator performance in Section VI. While the flexural resonators are shown to be inferior in terms of phase noise due to their low energy storage capability, the BAW resonators can provide performance close to the quartz resonators. The paper is concluded with Section VII where the impact of scaling on phase noise is analyzed.

\section{NONLINEAR OSCILLATIONS}

To characterize the nonlinear oscillatory motion and to estimate the maximum vibration amplitude, we review the results by Landau [3]. We take the bifurcation point as a measure of maximum usable vibration amplitude, as at higher vibration amplitudes, the oscillator trajectory depends on the initial conditions. Thus, the systems analyzed in this paper are weakly nonlinear and the analysis is restricted only to a single resonance excitation. Nonlinear effects can also lead to super and subharmonic resonances that can also limit the fundamental mode amplitude [4], [5].

The equation of motion for forced oscillations is

$$
m \ddot{x}+\gamma \dot{x}+k x=F(t)
$$

where $m$ is the lumped mass, $\gamma$ is the damping coefficient, $F(t)$ is the forcing term, and the nonlinear spring constant is $k=$ 


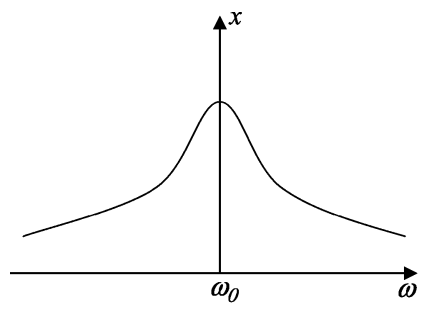

(a)

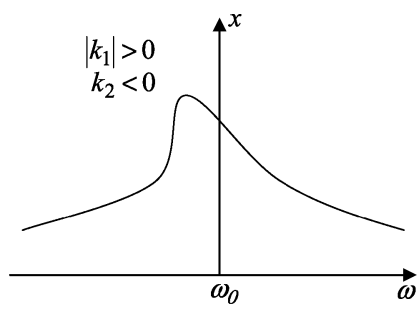

(b)

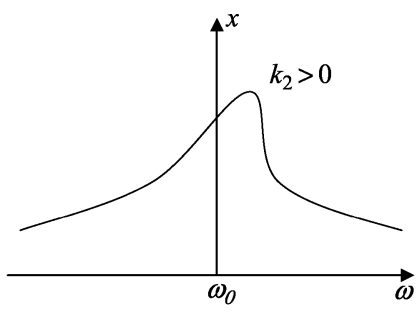

(c)

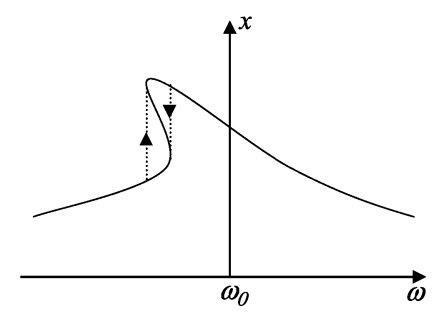

(d)

Fig. 1. The effect of anharmonic force on oscillator transmission curves. (a) Linear response. (b) $k_{1}$ and negative $k_{2}$ terms tilt resonance peak to a lower frequency. (c) Positive $k_{2}$ tilts resonance peak to a higher frequency. (d) Large amplitude vibrations result in hysteresis.

$k_{0}\left(1+k_{1} x+k_{2} x^{2}+\ldots\right)$, where $k_{0}$ is the linear term and $k_{1}$ and $k_{2}$ are the first- and second-order anharmonic terms. We also define the natural frequency $\omega_{0}=\sqrt{k_{0} / m}$ and quality factor $Q=\omega_{0} m / \gamma$. The quality factor is not usually defined for nonlinear systems but due to its information value for engineers, its use is justified for the weakly nonlinear systems analyzed here. The solutions to (1) can be obtained by the method of successive approximations by assuming a solution of form [3]

$$
x(t)=x_{0}+x_{1} \cos \omega t+x_{2} \cos 2 \omega t+x_{3} \cos 3 \omega t+\ldots .
$$

For vibrations without damping, the amplitude of the higher harmonics is given by

$$
\begin{aligned}
& x_{2}=\frac{k_{1}}{6} x_{1}^{2} \\
& x_{3}=\left(\frac{k_{1}^{2}}{48}+\frac{k_{2}}{32}\right) x_{1}^{3} .
\end{aligned}
$$

The resonance behavior changes in the presence of nonlinear terms and the resonance frequency is related to the vibration amplitude $x_{1}$ by

$$
\omega_{0}^{\prime}=\omega_{0}\left(1+\kappa x_{1}^{2}\right)
$$

where

$$
\kappa=\frac{3}{8} k_{2}-\frac{5}{12} k_{1}^{2} .
$$

This behavior is illustrated in Fig. 1. A typical linear amplitude vs. frequency curve is shown in Fig. 1(a). The first-order nonlinearity $k_{1}$ (positive or negative) causes tilting of the resonance peak to the left as shown in Fig. 1(b). A positive second-order nonlinearity results in tilting of the peak to a higher frequency as shown in Fig. 1(c). Increasing the excitation signal causes further increase in nonlinearity and eventually the transmission signal shows discontinuity due to frequency hysteresis (bifurcation) as demonstrated in Fig. 1(d).

The vibration amplitude at the point of bifurcation is

$$
x_{b}=\frac{1}{\sqrt{\sqrt{3} Q|\kappa|}} .
$$

As indicated in Fig. 2, the critical vibration amplitude (or the greatest vibration amplitude) is slightly higher than the vibration amplitude at the bifurcation point and is given by

$$
x_{c}=\frac{2}{\sqrt{3 \sqrt{3} Q|\kappa|}} .
$$

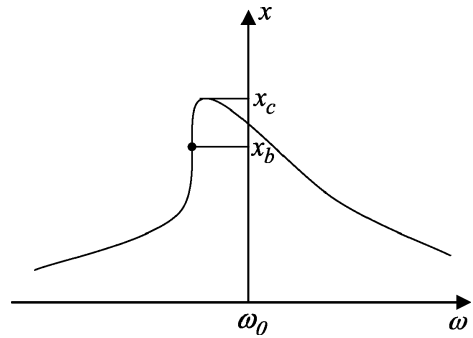

Fig. 2. The bifurcation point $x_{b}$ and critical vibration amplitude $x_{c}$.

If either $k_{1}$ or $k_{2}$ is dominant, the critical limit can be approximated from

$$
\begin{aligned}
& x_{c 1}=a_{1} \sqrt{\frac{1}{k_{1}^{2} Q}} \\
& x_{c 2}=a_{2} \sqrt{\frac{1}{\left|k_{2}\right| Q}}
\end{aligned}
$$

where $a_{1}=\sqrt{16 / 5 \sqrt{3}}$ and $a_{2} \sqrt{32 / 9 \sqrt{3}}$ (note correction to [2] for $a_{2}$ ).

We take the critical amplitude as the limit for mechanical energy storable in the resonator. Thus, the maximum stored energy is

$$
E_{m}^{\max }=\frac{1}{2} k_{0} x_{c}^{2}
$$

If all the linear device dimensions are scaled proportionally, the mechanical linear spring constant and the critical vibration amplitude scale as

$$
\begin{aligned}
k & \sim l \\
x_{c} & \sim \frac{l}{\sqrt{Q}}
\end{aligned}
$$

where $l$ is the linear device dimension and $Q$ is the quality factor. Thus, at the nonlinearity limit, the maximum energy stored scales as $E_{m}^{\max } \sim l^{3} / Q$. It can be seen that increasing the quality factor $Q$ reduces the amount of energy that can be stored in a resonator as the resonator becomes more susceptible to nonlinear effects.

\section{NONLINEARITIES IN MiCRORESONATORS}

The nonlinearities in electrostatically actuated resonators can have mechanical and capacitive origin. The mechanical nonlinearity is due to geometrical and material effects in the resonating element while the capacitive nonlinearity is due to electrostatic 


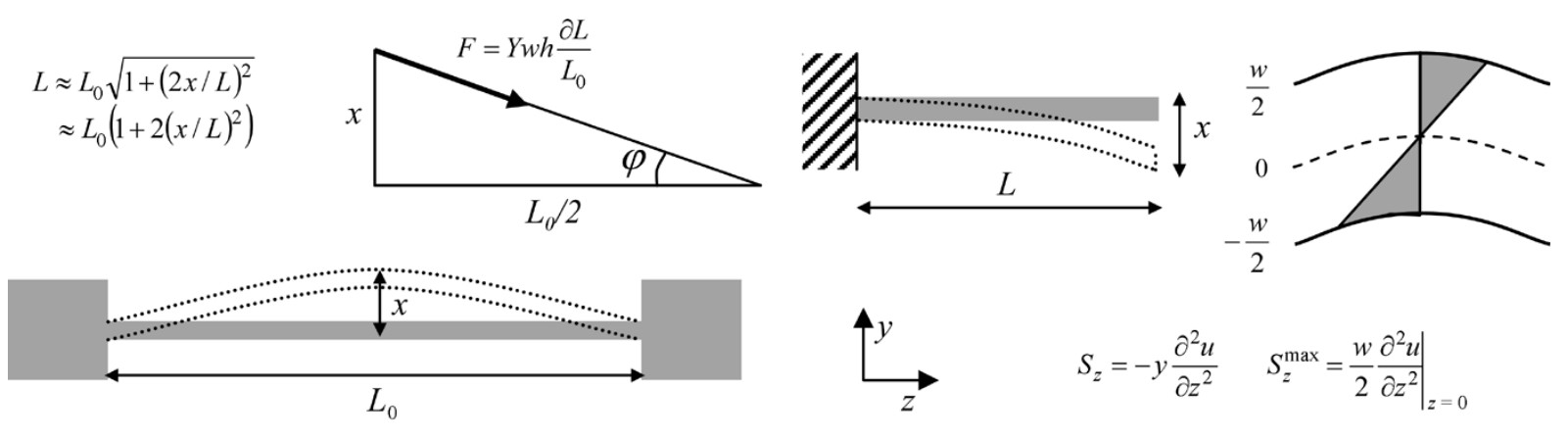

(a)

(b)

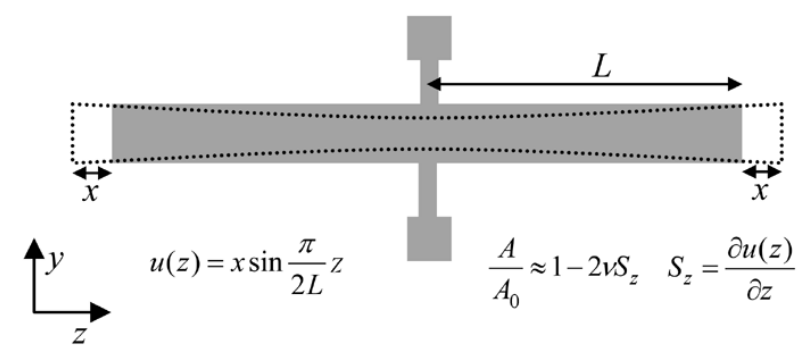

(c)

Fig. 3. Comparison of three different microresonators. (a) Spring hardening due to stretching of clamped-clamped beam (bridge resonator). (b) Vibrations of clamped-free beam (cantilever). (c) Spring softening due change in BAW resonator area.

coupling mechanism. In this section, both effects are considered and approximate analysis is carried out.

\section{A. Mechanical Nonlinearity}

It is illustrative to estimate the mechanical nonlinearity for three devices, a bridge, a cantilever, and a bulk acoustic wave (BAW) resonator, shown in Fig. 3. Each resonator is sized to have the natural frequency of $13 \mathrm{MHz}$. The aim of this analysis is to get an order-of-magnitude comparison of the maximum energy limit. For clarity, only geometrical effects are considered and the accurate analysis that includes material effects is postponed until Section IV.

1) Bridge Resonator: Fig. 3(a) shows a bridge resonator, also known as the clamped-clamped beam resonator. According to the classical beam theory, the first mode shape is

$$
u(z)=x C[\sinh \beta z-\sin \beta z-\alpha(\cosh \beta z-\cos \beta z)]
$$

where $x$ is the vibration amplitude at the beam center and the constants are $C \approx 0.619, \beta L \approx 4.730$, and $\alpha \approx 1.018$ [6]. Assuming a point force excitation at the bridge center, the lumped effective mass and the spring constant for the first resonance are

$$
\begin{aligned}
m & =0.396 \cdot \rho w h L_{0} \\
k_{0} & =125.1 \cdot \frac{Y I}{L_{0}^{3}}
\end{aligned}
$$

where $I$ is the moment of inertia $I=h w^{3} / 12, Y$ is the Young's modulus, $h$ is the beam height, $w$ is the beam width, and $L_{0}$ is the beam length. Large deformations result in an additional anharmonic force due tension caused by the change in the beam length.

To obtain a rough estimate for the anharmonic term, the displacement profile is approximated with a triangle as shown in
Fig. 3(a). While the triangle is rather crude approximation to the mode shape given by (11), it allows easy order of magnitude estimation of the nonlinear spring force. The beam tensioning due to the elongation is $F=Y w h \delta L / L_{0}$ and the resulting force in $x$-direction is

$$
F_{x}=2 F \sin \varphi \approx \frac{8 Y w h x^{3}}{L_{0}^{3}}=\frac{0.767 k_{0} x^{3}}{w^{2}} .
$$

Thus, the nonlinear mechanical spring is

$$
\begin{aligned}
k(x) & =k_{0}\left(1+k_{2} x^{2}\right) \\
k_{2} & =\frac{0.767}{w^{2}} .
\end{aligned}
$$

Finite element analysis shows that this simple estimate is accurate within $30 \%$ for a typical bridge microresonator. The critical vibration amplitude given by (8) is $x_{c 2}=65 \mathrm{~nm}$ for a $13 \mathrm{MHz}$ bridge resonator with $Q=10000$ and dimensions of $w=4 \mu \mathrm{m}, h=10 \mu \mathrm{m}$, and $L=52 \mu \mathrm{m}$. The corresponding maximum stored energy is $E_{m}^{\max }=2.6 \cdot 10^{-11} \mathrm{~J}$.

2) Cantilever Resonator: Unlike the bridge resonator, the cantilever resonator shown in Fig. 3(b) has no single dominant nonlinear effect [7]. While a full nonlinear analysis is beyond the scope of this paper, it is useful to obtain an upper limit for the stored vibration energy for scaling and comparison purposes. Given that the nonlinear effects are weak, we optimistically assume that the resonator can be driven close to the silicon fracture point.

According to the linear beam theory, the effective mass and the spring constant are

$$
\begin{aligned}
m & =0.250 \cdot \rho w h L \\
k_{0} & =3.091 \cdot \frac{Y I}{L^{3}}
\end{aligned}
$$


for a point force excitation at the cantilever end [6]. The mode shape for linear vibrations is

$$
u(z)=x C[\sin \beta z-\sinh \beta z+\alpha(\cosh \beta z-\cos \beta z)]
$$

where $x$ is the vibration amplitude at the beam end and the constants are $C \approx 0.367, \beta L \approx 1.875$, and $\alpha \approx 1.362$. The maximum strain is at the anchor point and is given by

$$
S_{z}^{\max }=\left.\frac{w}{2} \frac{\mathrm{d}^{2} u(z)}{\mathrm{d} z^{2}}\right|_{z=0}
$$

The typical fracture strain for bulk micromachined silicon cantilevers is $1 \cdot 10^{-2}$ [8]. Assuming that the beams can be vibrated at $50 \%$ of the fracture point, a $13 \mathrm{MHz}$ resonator with dimensions of $h=10 \mu \mathrm{m}, w=4 \mu \mathrm{m}$, and $L=20.5 \mu \mathrm{m}$ has a maximum vibration amplitude of $300 \mathrm{~nm}$. The corresponding maximum stored energy is $E_{m}^{\max }=1.5 \cdot 10^{-10} \mathrm{~J}$.

3) BAW Resonator: The beam BAW resonator shown in Fig. 3(c) demonstrates a geometrical spring softening effect due to the change in the cross sectional beam area. The wave equation for a longitudinal mode in beam is

$$
\rho A_{0} \frac{\partial^{2} u}{\partial t^{2}}=Y \frac{\partial}{\partial z}\left(A\left(S_{z}\right) \frac{\partial u}{\partial z}\right)
$$

where $A_{0}$ is the undeformed beam cross sectional areas and the deformed area $A\left(S_{z}\right)$ is given by

$$
A \approx A_{0}\left(1-2 \nu S_{z}\right)=A_{0}\left(1-2 \nu \frac{\partial u(z, t)}{\partial z}\right)
$$

where $\nu$ is the Poisson's ratio. The solution to (18) is approximated by the linear solution

$$
u(z, t)=\frac{x(t) \sin \pi z}{2 L}
$$

where $x$ is the motion of the beam tip [2]. Substituting (20) into (18) and integrating over the mode shape leads to

$$
\rho A_{0} L \frac{\partial^{2} x}{\partial t^{2}}=\frac{\pi^{2}}{4} \frac{A_{0} Y}{L} x-\frac{\pi^{2}}{3} \frac{\nu A_{0} Y}{L^{2}} x^{2} .
$$

The effective mass and the nonlinear spring constants can be recognized as

$$
\begin{aligned}
& m=\rho A L \\
& k_{0}=\frac{\pi^{2} A_{0} Y}{4 L} \quad \text { and } \quad k_{1}=-\frac{4 \pi \nu}{3 L} .
\end{aligned}
$$

Using a typical values of $Q=100000, A_{0}=100 \mu \mathrm{m}^{2}$, and $L=160 \mu \mathrm{m}$ for a $13 \mathrm{MHz}$ BAW resonator [2], the critical vibration amplitude given by (8) is $x_{c 1}=500 \mathrm{~nm}$ and the corresponding maximum energy is $E_{m}^{\max }=3.7 \cdot 10^{-8} \mathrm{~J}$.

4) Microresonator Comparison: Comparing the different resonators, the BAW resonator is seen to have orders-of-magnitude larger energy storage capacity $\left(E_{m}^{\max }=3.7 \cdot 10^{-8} \mathrm{~J}\right)$ than the bridge $\left(E_{m}^{\max }=2.6 \cdot 10^{-11} \mathrm{~J}\right)$ and cantilever resonator $\left(E_{m}^{\max }=1.5 \cdot 10^{-10} \mathrm{~J}\right)$ operating at the same frequency. Normalizing the stored energy with the resonator volume $V$ gives the maximum energy density $E_{m}^{\max } / V$. For the bridge, cantilever, and BAW resonator this is $1.3 \cdot 10^{4} \mathrm{~J} / \mathrm{m}^{3}, 1.9 \cdot 10^{5} \mathrm{~J} / \mathrm{m}^{3}$, and $2.1 \cdot 10^{6} \mathrm{~J} / \mathrm{m}^{3}$, respectively. Thus, the high energy storage capability of the BAW resonators arises from two factors: the high maximum energy density and the large volume.

The approximate analysis in this section thus indicates that the BAW devices are candidates for large energy storage resonators. As the maximum energy density is of fundamental interest, the approximate analysis for the BAW resonators will be refined in Section IV where the material effects are also considered.

\section{B. Capacitive Nonlinearity}

Due to inverse relationship between displacement and parallel plate capacitance, electrostatic coupling introduces nonlinear forcing terms [9]. The nonlinear spring constants are obtained by a series expansion of the electrostatic force

$$
\begin{aligned}
& F=-\frac{U_{\mathrm{dc}}^{2}}{2} \frac{\partial C}{\partial x} \\
& C=\frac{\epsilon_{0} A_{e}}{d-x}
\end{aligned}
$$

where $U_{\mathrm{dc}}$ is the bias voltage, $A_{e}$ is the electrode area, $\epsilon_{0}$ is the permittivity of free space, $d$ is the electrode gap, and $x$ is the resonator displacement [2]. Including the terms up to the second-order gives

$$
\begin{aligned}
k(x) & =k_{0 e}\left(1+k_{1 e} x+k_{2 e} x^{2}\right) \\
k_{0 e} & =-\frac{U_{\mathrm{dc}}^{2} \epsilon_{0} A_{e}}{d^{3}}, \quad k_{1 e}=\frac{3}{2 d}, \quad \text { and } \quad k_{2 e}=\frac{2}{d^{2}} .
\end{aligned}
$$

The second-order correction can be shown to be the dominant nonlinear electrostatic term [2]. Since the electrical spring coefficient is proportional to $U_{\mathrm{dc}}^{2}$, the capacitive nonlinearity can be reduced by lowering the bias voltage. Also, the nonlinearity could be significantly reduced with different electrode configuration, e.g., comb-drive actuation. Thus, electrostatic nonlinearity, while inherent to actuation mechanism, does not set a fundamental limit to the vibration amplitude. In practice, for resonators such as the cantilever in Section III-A2 that have low mechanical stiffness, the capacitive nonlinearity can be significant. As an example, assuming electrode area $A_{e}=100 \mu \mathrm{m}^{2}$, gap $d=0.5 \mu \mathrm{m}$, and bias voltage $U_{\mathrm{dc}}=50 \mathrm{~V}$, gives hysteresis limit of $x_{c 3 e}=57 \mathrm{~nm}$ for the cantilever resonator. This can be compared to the $300 \mathrm{~nm}$ estimate for the mechanical limit.

In addition to the nonlinear spring effects, the capacitive coupling results in distortion of the motional current. These harmonics can be calculated from

$$
i=U_{\mathrm{dc}} \frac{\partial C}{\partial t}=-U_{\mathrm{dc}} \frac{\epsilon_{0} A_{e}}{d^{2}}\left(1-2 \frac{x}{d}+3\left(\frac{x}{d}\right)^{2}+\ldots\right) \frac{\partial x}{\partial t} .
$$

Thus, even linear vibrations can result in harmonics and the harmonics due to capacitive coupling can be much larger than the harmonics arising from the nonlinearity and given by (3). 


\section{MECHANICAL NONLINEARITY IN SiliCON BAW RESONATORS}

As was shown in Section III-A3, the geometrical nonlinearities may be very small for the BAW resonators and material effects have to be included in the analysis. In this section, an accurate analysis of mechanical nonlinearity is presented for the BAW resonators. First, the nonlinear Young's modulus is calculated for bulk silicon. Next, a model that incorporates the material nonlinearity is presented and nonlinear vibrations are simulated using the method of harmonic balance. The simulations are compared to experimental results obtained for two types of BAW resonators: longitudinal mode beam resonator and extensional mode plate resonator shown in Fig. 5.

\section{A. Theory of Large Deformations}

The Cauchy stress due to finite deformation including the geometrical (area and volume change) and material stiffness effects is

$$
\sigma_{i j}(X)=\frac{\rho_{X}}{\rho_{a}} \frac{\partial X_{i}}{\partial a_{k}} \frac{\partial X_{j}}{\partial a_{k}}\left(c_{i j k l} \eta_{k l}+c_{i j k l m n} \eta_{k l} \eta_{m n}\right)
$$

where $X$ is the particle coordinate at finite deformation, $a$ is the undeformed state, $\rho_{X}$ and $\rho_{a}$ are the deformed and undeformed densities, $c_{i j l k}$ and $c_{i j k l m n}$ are the second and third-order stiffness tensors, and $\eta_{k l}$ is the Lagrangian strain [10]. The thirdorder stiffness tensor for silicon has been experimentally obtained using ultrasonic wave measurements [11] and theoretical analysis [12]. This data and (26) enable computation of nonlinear strain dependent engineering Young's modulus

$$
Y=\frac{T}{S}=Y_{0}\left(1+Y_{1} S+Y_{2} S^{2}\right)
$$

where $T$ is the force divided by the initial undeformed area (engineering stress), $S=\partial u / \partial x$ is the displacement gradient with respect undeformed coordinates (engineering strain), and $Y_{1}$ and $Y_{2}$ are the first- and second-order corrections respectively. Calculated values for the nonlinear Young's modulus are tabulated in Table I with values in [100]-direction agreeing with the published analytical results in [10]. The contribution of the anharmonic stiffness tensor $c_{i j k l m n}$ is found to be significant. For example, accounting only for the geometric effects gives $Y_{1}=1.5$ and $Y_{2}=0.5$ for the [100] beam extension. The third-order stiffness tensors in [11] and [12] are slightly different but the resulting difference in the nonlinear Young's modulus is only about $5 \%$.

Unfortunately, no information exists on the effect of doping on the anharmonic stiffness tensor. Thus, the calculated values may not be accurate for the highly boron doped silicon $\left(N_{B} \approx\right.$ $\left.5 \cdot 10^{18} 1 / \mathrm{cm}^{3}\right)$ used for the microresonators measured in this study. Nevertheless, the literature data allows a comparison of measured resonator nonlinearities and the fundamental material limits.

The relationship between the nonlinear Young's modulus and spring constants is

$$
\begin{aligned}
k(x) & =k_{0}\left(1+k_{1} x+k_{2} x^{2}\right) \\
k_{0} & =\frac{A Y_{0}}{L}, \quad k_{1}=\frac{Y_{1}}{L}, \quad \text { and } \quad k_{2}=\frac{Y_{2}}{L^{2}}
\end{aligned}
$$

TABLE I

CAlculated Nonlinear ENGineERING Young's Modulus

\begin{tabular}{lrrr}
\hline & $Y_{0}[\mathrm{GPa}]$ & $Y_{1}$ & $Y_{2}$ \\
\cline { 2 - 4 } beam ([100]) & 130 & 0.65 & -4.6 \\
plate extension & 181 & -2.8 & -8.3 \\
\hline
\end{tabular}

where $x$ is the spring stretching, $A$ is the area, and $L$ is the length. We emphasize that this includes both the material and geometrical effects. Using (8) and (28), the critical strain amplitude at the hysteresis due to first- and second-order mechanical nonlinearity corrections can be approximated. Based on the computed values for Young's modulus in Table I, we estimate the critical vibration amplitude $x_{c} \approx 180 \mathrm{~nm}$ for a plate resonator in Fig. 5 with $L=160 \mu \mathrm{m}$. In this case, both the firstand second-order correction terms are significant.

\section{B. Modeling of the Distributed Nonlinearity}

To accurately simulate the nonlinear vibrations, the distributed nature of stress and strain has to be included in the model. In our devices the resonator modal shape is to a good approximation sinusoidal and the strain is the highest at the center. A full distributed model would be computationally very demanding and therefore the continuum is approximated with a discrete chain of masses connected with nonlinear springs. As shown in Fig. 4, a good approximation is obtained with a relatively small number of masses. A four mass system appears to be a good compromise between the accuracy and simulation speed, and is used in this paper. The mass-spring chain model has been implemented as an electrical-equivalent model in the Aplac simulation software. In addition to the mechanical nonlinearity, the equivalent circuit includes an accurate model of the capacitive coupling [9]. Displacement versus frequency responses to a sinusoidal excitation are simulated using the harmonic balance analysis [13]. As the harmonic balance analysis is carried out in the frequency domain, it is computationally efficient for systems that have a high quality factors and are thus slow to settle in the time domain (transient) analysis.

\section{Measured Nonlinear Vibrations}

To characterize the nonlinear vibrations in single-crystal silicon micromechanical resonators, two bulk acoustic wave (BAW) resonator designs shown in Fig. 5 were measured. The devices were fabricated by etching a SOI wafer. Both the beam and plate BAW resonators show high quality factors exceeding 100000 and operate at $11.7 \mathrm{MHz}$ and $13.1 \mathrm{MHz}$ respectively. Further details of these resonators are provided in [2] and [14].

The measurements were done using a HP4195A network analyzer and the resonance signal was buffered with a JFET preamplifier with a low $100 \Omega$ input impedance to rule out resonator loading by the measurement set-up. Fig. 6 shows the measured and simulated transmission amplitudes $\left|S_{21}\right|$ for the plate BAW device shown in Fig. 5. At higher drive levels, the resonator peak becomes sharper and shifts down in frequency. As discussed in Section III-A, this tilting is expected with $k_{1}$ and/or negative $k_{2}$.

The measured and simulated data shown in Fig. 6(a) correspond to the best fit values $Y_{1}=-1.4$ and $Y_{2}=-4.0$. These experimentally obtained values are about $50 \%$ lower than theoret- 

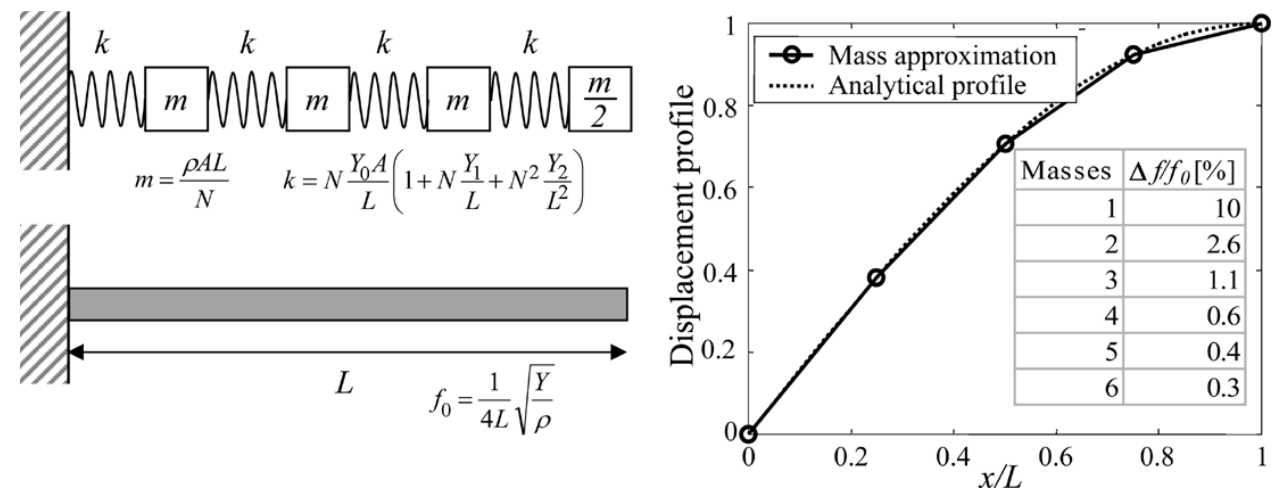

Fig. 4. Equivalent mechanical model used in Aplac circuit simulator. Material nonlinearity is included as nonlinear springs. As the number of discrete elements $N$ is increased, the frequency difference $\Delta f / f_{0}$ between discrete and continuum model approaches zero.
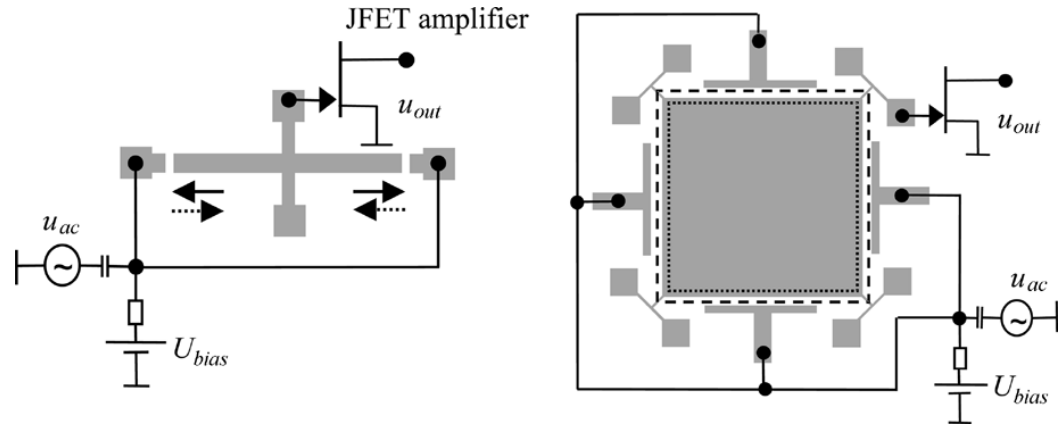

Fig. 5. Schematics of the beam and plate resonators used in measuring nonlinearities in single-crystal BAW resonators.

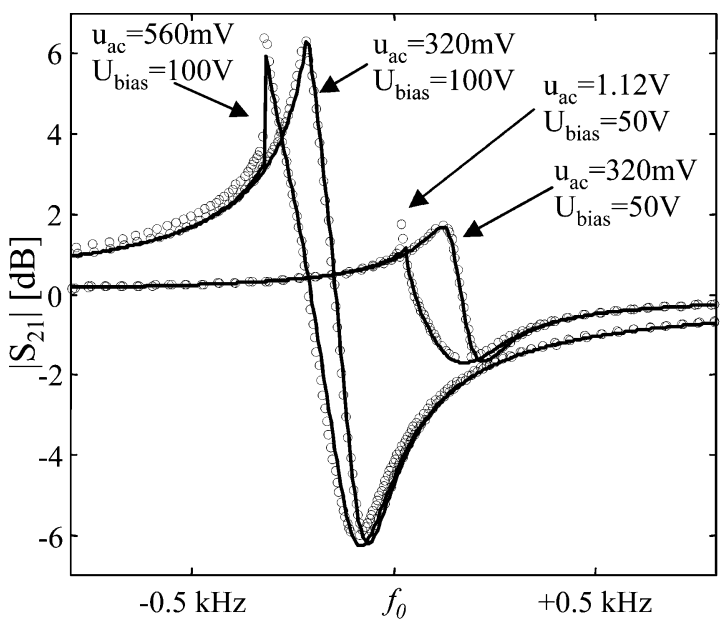

(a)

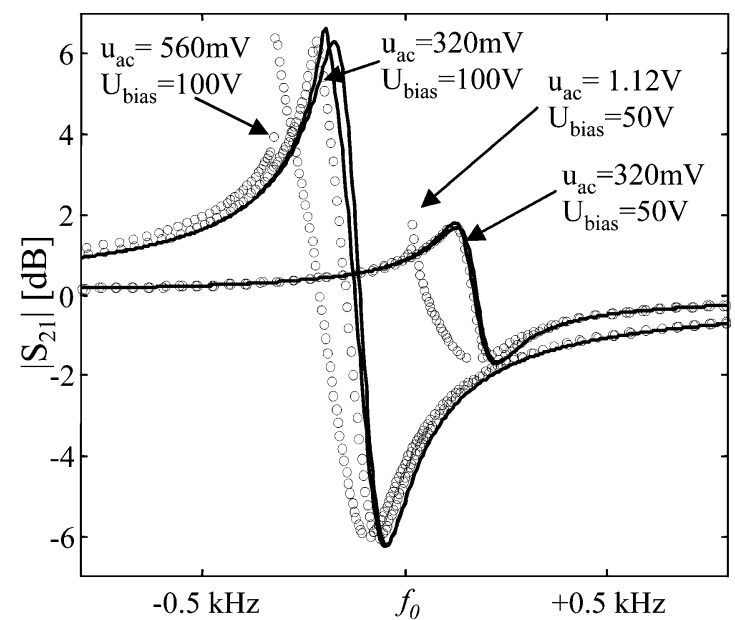

(b)

Fig. 6. Measured and simulated transmission curves for 2-D plate $\left(f_{0}=13.1 \mathrm{MHz}\right)$ with nonlinear capacitive and mechanical effects. The maximum vibration amplitude at the hysteresis limit $x_{C}$ was $155 \mathrm{~nm}$ independent of bias showing that hysteresis limit is due to mechanical and not capacitive nonlinearity. (a) Measured and simulated (-) transmission $\left|S_{21}\right|$ curves with the material nonlinearity included in the model $\left(Y_{1}=-1.4\right.$ and $\left.Y_{2}=-4.0\right)$. The highest excitation level results in a discontinuity (the sweep direction is from right to left). (b) Measured (o) and simulated (-) transmission $\left|S_{21}\right|$ curves without the material nonlinearity included in the model. Capacitive spring softening alone does not explain the frequency shift.

ically estimated for a solid plate. This discrepancy can probably be attributed to the etch holes $(39 \times 39$ matrix of $1.5 \mu \mathrm{m}$ holes $)$ in the plate that lower the effective Young's modulus. Another source of discrepancy may the corner anchoring that although flexible may add to the nonlinearity. Nevertheless, the obtained maximum vibration amplitude is close to the theoretical limit for bulk silicon. The hysteresis limit was $x_{c}=155 \mathrm{~nm}$, which corresponds to average strain of $S_{x}=S_{y}=9.7 \cdot 10^{-4}$ across the resonator and maximum strain of $S_{x}^{\max }=S_{y}^{\max }=1.5 \cdot 10^{-3}$ at the resonator center. This corresponds to the stored energy of $190 \mathrm{~nJ}$ or average energy density of $E_{m} / V=1.9 \cdot 10^{5} \mathrm{~J} / \mathrm{m}^{3}$.

To quantify the effect of nonlinearity due to the capacitive coupling and to show that the nonlinearity is indeed of mechanical origin, the transmission was also simulated without the mechanical nonlinearity in the model. As the capacitive nonlinearity given by (24) increases as a square of bias voltage, it is insignificant at low bias voltages but becomes important at high bias voltages. This is evident in Fig. 6(b), where simulation 


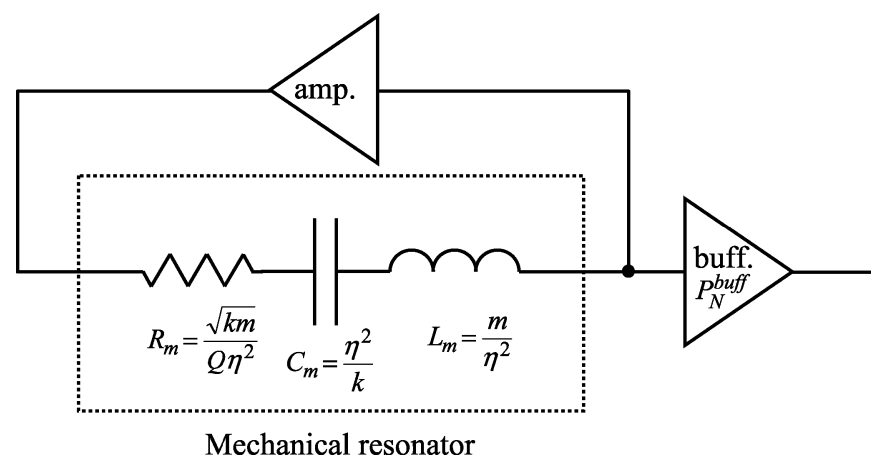

Fig. 7. The electrical equivalent circuit for MEMS-based oscillator.

without mechanical nonlinearity show no excitation amplitude dependence at $50 \mathrm{~V}$ bias voltage. Higher bias voltages show capacitive spring softening effect, but even at $U_{\text {bias }}=100 \mathrm{~V}$ this is not enough to explain the observed nonlinearity. A further proof that the mechanical nonlinearity dominates at low bias voltages is obtained by looking at the bias and excitation voltage product $U_{\text {bias }} \cdot u_{\text {ac }}$ at the hysteresis limit. As the driving force and consequently the vibration amplitude is proportional to $U_{\text {bias }} \cdot u_{\text {ac }}$ this remains constant if the mechanical nonlinearity dominates. Our measurements for the plate BAW resonator indeed show that hysteresis is obtained at constant $U_{\text {bias }} \cdot u_{\mathrm{ac}}$.

As both the first- and second-order nonlinearity can cause similar distortion on the transmission curve, there is uncertainty about the relative contribution of $Y_{1}$ and $Y_{2}$. In principle the first- and second-order effects can be differentiated by looking at the vibration spectrum. Unfortunately, as discussed in Section III-B, the parallel plate transducer produces harmonics even for linear vibrations. For our devices, the harmonics in motional current due to capacitive coupling given by (25) are orders-of-magnitude larger than the harmonics due nonlinear vibrations given by (3). Thus, measuring the motional current spectrum does not yield further information about the mechanical nonlinearity. Future work will measure the mechanical vibrations using optical interferometric techniques.

The measured beam BAW's showed similar behavior but the measured values showed larger variation from device to device. We attribute this to the larger surface-to-volume ratio that causes small geometrical or surface defects to have a larger effect. Also, the mechanical spring constant for the beams is much smaller than for the plate causing the capacitive nonlinearity to be more significant. With capacitive nonlinearity shadowing the mechanical nonlinearity, accurate absolute values for the nonlinear mechanical spring constant could not be obtained. Based on our measurements, we estimate upper limits of $\left|Y_{1}\right| \leq 3$ and $\left|Y_{2}\right| \leq 10$ for the correction terms. Thus, even for the 1-D beam BAW's, we can conclude that the measured mechanical nonlinearities are not significantly larger than estimated from the theory and that the devices can be operated near the fundamental strain limit.

\section{Nonlinearity and Phase Noise Performance}

To show the importance of nonlinearities for microresonator performance, it is useful to consider noise-to-carrier ratio in an oscillator. Fig. 7 shows an electrical equivalent circuit for a MEMS resonator connected in an oscillator loop with a loop amplifier and a buffer to interface with the outside world. The schematic representation that uses two amplifiers is chosen as it simplifies the analysis by the separating of the near-carrier mechanical noise and the far-from-carrier amplifier noise (noise floor).

The motional resistance $R_{m}$, capacitance $C_{m}$, and inductance $L_{m}$ depend on the effective spring constant $k$, the effective mass $m$, the quality factor $Q$, and the electromechanical transduction factor $\eta$ [2]. The relation between current and mechanical velocity is $i=\eta \dot{x}$.

The mechanical vibration energy stored in the resonator is

$$
E_{m}=\frac{1}{2} k x_{c}^{2}=L_{m} \overline{i_{\text {sig }}^{2}}
$$

where $\overline{i_{\text {sig }}^{2}}$ is the mean-square signal current through the circuit. The theoretical maximum power deliverable to the buffer is the same as the power dissipated in the motional resistance i.e.

$$
P_{\text {sig }}=R_{m} \overline{i_{\text {sig }}^{2}}=\frac{\omega E_{m}}{Q} .
$$

In specifying quartz crystal oscillators, the $P_{\text {sig }}$ and $i_{\text {sig }}$ are interchangeably referred to as the "drive level" and is a measure of resonator power handling capacity.

The noise current due to motional resistance $R_{m}$ at the buffer input is shaped by $R L C$ impedance $Z(\omega)$ and is

$$
\overline{i_{n}^{2}}=\frac{4 k T R_{m}}{|Z(\omega)|^{2}}
$$

For a frequency offset $\Delta \omega$ from the center frequency $\omega_{0}$, the impedance of the series $R L C$-circuit is

$$
Z(\Delta \omega) \approx i 2 L \Delta \omega
$$

We can thus write the noise power density due to mechanical losses at the buffer input as

$$
P_{N}^{\text {mech }}=4 k T\left(\frac{\omega_{0}}{2 Q \Delta \omega}\right)^{2} .
$$

The loop amplifier will also add noise but with proper noise optimization it's contribution to near-carrier noise can be made small. For simplicity, the loop amplifier noise is therefore omitted here.

The buffer amplifier noise sets a fundamental limit for the oscillator performance at large frequency offsets. The resonator impedance seen by the buffer amplifier is a rapidly varying function of $\Delta \omega$, and thus perfect noise matching cannot be obtained for all frequency offsets. In practice, the consequence is the constant amplifier noise floor at large frequency offsets. We model the buffer noise using a white noise power spectral density $P_{N}^{\text {buff }}$.

Adding the buffer noise $P_{N}^{\text {buff }}$ and dividing (33) by two to account only the phase not amplitude noise gives the overall phase noise spectrum. It is customary to normalize this with the carrier power $P_{\text {sig }}$ to obtain the phase noise-to-carrier ratio

$$
\mathcal{L}(\Delta \omega)=\frac{2 k T}{P_{\text {sig }}}\left(\frac{\omega_{0}}{2 Q \Delta \omega}\right)^{2}+\frac{P_{N}^{\text {buff }}}{2 P_{\text {sig }}} .
$$


Thus, we have a near-carrier region originating from mechanical dissipations where noise falls as $1 / \Delta \omega^{2}$, and a constant noise floor region dominated by the buffer amplifier. Equation (34) bears close similarity to the generally used models for oscillator phase noise (e.g., "Leeson's equation") [15], [16]. The analysis has ignored the effect of $1 / f$-noise, which would result in noise falling as $1 / \Delta \omega^{3}$ very close to the carrier.

The important observation is that both terms in (34) have an inverse dependence of signal power $P_{\text {sig }}$ and consequently of the energy $E_{m}$ (30). As was shown in Section II, the maximum energy at nonlinear limit scales with device size as $E_{m}^{\max } \sim$ $l^{3} / Q$. Thus, assuming fixed frequency, the signal power scales $P_{\text {sig }} \sim l^{3} / Q^{2}$ and the resonator noise floor at nonlinear limit is proportional to $Q^{2} / l^{3}$. Thus, the energy storage capacity sets the fundamental performance limit for microresonators and the small size cannot be compensated with a high-quality factor.

Finally, we note that the electromechanical transduction factor $\eta$ does not appear in (34) as it assumes optimal power coupling. However, in practice a sufficient $\eta$ is required to optimally realize the oscillator using technologically feasible amplifier interface.

\section{PHASE NOISE COMPARISON}

Using (34), the theoretical phase noise can be compared for the microresonators analyzed in this paper and for a macroquartz crystal. The bifurcation limit for a $5 \mathrm{MHz}$ AT-cut quartz crystal resonator $\left(Q=800000, R_{m}=165 \Omega\right)$ is $i_{\max }=2.1 \mathrm{~mA}$ and the corresponding maximum drive level and stored energy are $P_{\text {sig }}=(1 / 2) R_{m} i_{\max }^{2}=360 \mu \mathrm{W}$ and $E_{m}^{\max }=P Q / \omega=9.3 \mu \mathrm{J}$, respectively [17]. These published values roughly agree with our own measurements of the hysteresis in quartz crystals. The crystal volume is estimated to be $200 \mathrm{~mm}^{3}$ and the corresponding stored energy density is $E_{m} / V=50 \mathrm{~J} / \mathrm{m}^{3}$. Extrapolated to $13 \mathrm{MHz}$ (see Section VII), the critical parameters are $Q=300000, P_{s i g}=360 \mu \mathrm{W}$, $E_{m}^{\max }=1.4 \mathrm{~J} / \mathrm{m}^{3}$, and $E_{m} / V=190 \mathrm{~J} / \mathrm{m}^{3}$. Comparison to the silicon plate BAW resonator $\left(E_{m} / V=1.8 \times 10^{5} \mathrm{~J} / \mathrm{m}^{3}\right)$ shows that orders-of-magnitude higher energy density can be achieved with silicon micromechanical resonators than with shear-mode macroscopic quartz devices. This can partially compensate for the small size of RF-MEMS oscillators.

Fig. 8 shows the theoretical phase noise density for the macro AT quartz crystal, silicon bridge, silicon cantilever, and silicon beam BAW and plate BAW resonators summarized in Table II. All but the cantilever resonators are assumed to be driven to the mechanical hysteresis limit. The cantilever resonator operates at $50 \%$ of the fracture limit (see Section III-A2). In the plots, we have assumed buffer noise of $P_{N}^{\text {buff }}=-155 \mathrm{dBm} / \sqrt{\mathrm{Hz}}$ (or $\bar{v}_{n}=4 \mathrm{nV} / \sqrt{\mathrm{Hz}}$ in $50 \Omega$ system). The bridge and cantilever resonators' poor noise performance is due to low quality factor and energy storage capability. In comparison, the beam BAW has improved performance but the noise floor is still about $30 \mathrm{~dB}$ higher than for macro quartz crystal oscillator. The plate BAW resonator has the same quality factor as the beam BAW but due to the improved energy storage capability, the noise performance is close to the quartz crystal. In practice the oscillators must be operated well below the bifurcation to avoid aliasing of noise and the bifurcation limit is used for comparison purpose only and it may not be reached with real oscillators.

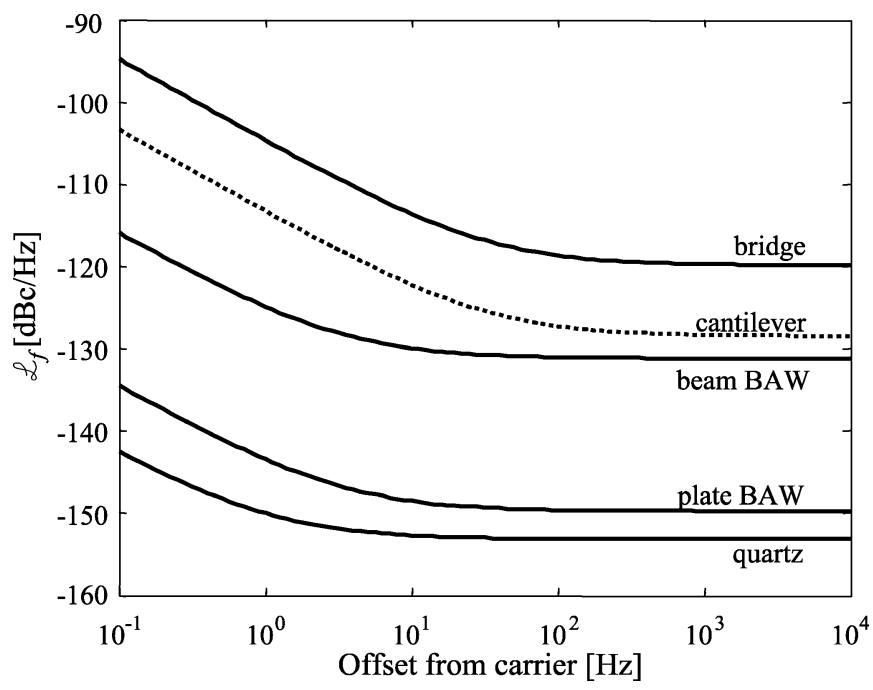

Fig. 8. Comparison of theoretical phase noise for a bridge, beam-BAW, 2-D-BAW, and macroscopic quartz resonator based oscillators.

It may be of interest to compare the noise analysis presented here to the analysis on noise and scaling in [18]. The devices analyzed here are much larger and consequently the noise due to internal dissipation is much larger than the quantum noise sources in [18]. However, when scaling to smaller dimensions, the quantum effects can be significant.

For a real world performance comparison, Fig. 9 shows measured single-sideband (SSB) phase noise to carrier ratio for a test oscillator based on the plate resonator [19]. The oscillator demonstrates that sufficient energy can be stored in the microresonator to satisfy the GSM-specifications for phase noise.

\section{SCALING TO HighER FREQUENCIES}

This paper has focused on $13 \mathrm{MHz}$ resonators - a frequency typically used for reference oscillator applications. In transreceiver, the reference frequency is multiplied by a factor $\alpha$ to generate the local oscillator (LO) at the carrier frequency (typically 1-2 GHz). Due to this frequency multiplication, the phase noise scales as $\alpha^{2}$ [16]. Alternatively, the resonator can be scaled to operate at higher frequency to obtain the LO frequency directly. Thus, it is of interest to develop a scaling law for micro-oscillator phase noise as a function of resonator natural frequency.

Assuming that the frequency-quality factor product $f \cdot Q$ is constant [20], the device scales as

$$
\begin{aligned}
\omega_{0}^{\prime} & =\alpha \omega_{0} \\
l^{\prime} & =\frac{l}{\alpha} \\
Q^{\prime} & =\frac{Q}{\alpha} \\
E_{m}^{\prime} & =\frac{E_{m}}{\alpha^{2}} \\
P_{\text {sig }}^{\prime} & =P_{\text {sig }}
\end{aligned}
$$

where all the device dimension are scaled by the same factor $\alpha$ and the device is operated at the hysteresis limit. The phase noise for the scaled device is

$$
\mathcal{L}(\Delta \omega)=\alpha^{4} \frac{2 k T}{P_{\text {sig }}}\left(\frac{\omega_{0}}{2 Q \Delta \omega}\right)^{2}+\frac{P_{N}^{\text {buff }}}{2 P_{\text {sig }}} .
$$


TABLE II

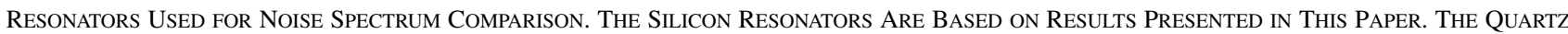
RESONATOR IS EXTRAPOLATED FROM DATA FOR 5 MHz AT-CUT QUARTZ CRYSTAL [17]

\begin{tabular}{lrrrrr}
\hline & Quartz & Bridge & Cantilever & Beam BAW & Plate BAW \\
\cline { 2 - 6 } Quality factor & 300000 & 10000 & 10000 & 100000 & 100000 \\
Stored energy[nJ] & 1400 & 0.026 & 0.14 & 2.6 & 180 \\
Energy density[J/m $\left.{ }^{3}\right]$ & 190 & $1.3 \cdot 10^{4}$ & $1.5 \cdot 10^{5}$ & $8.0 \cdot 10^{4}$ & $1.8 \cdot 10^{5}$ \\
\hline
\end{tabular}

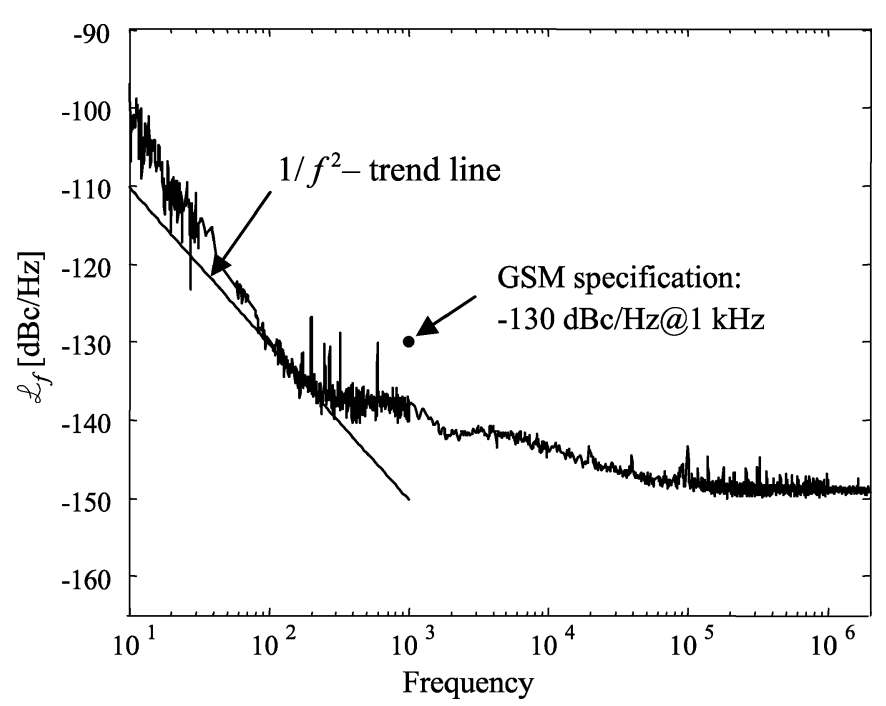

Fig. 9. Measured noise spectrum for a microresonator-based low-phase noise oscillator.

The noise floor is seen to remain constant while the near carrier noise degrades as $\alpha^{4}$. In practice, the noise floor will deteriorate for higher frequency oscillator as it is more difficult to obtain optimal coupling. Thus, from purely phase noise considerations, it is better to use low frequency reference and multiply it to higher frequency than it is to make a high frequency oscillator [16].

\section{CONCLUSION}

For many practical applications, the resonator power handling capacity and quality factor are equally important. In this paper the nonlinear limits for silicon resonators have been quantified and expressions for scaling of resonator energy storage and power handling capacity were derived. Different microresonators were analyzed using one degree-of-freedom model with anharmonic spring forces. The maximum vibration amplitude was estimated from bifurcation in the vibration amplitude vs. frequency curve. Increasing the resonator quality factor was shown to make the resonator more susceptible to nonlinearities and lower the maximum energy stored. The geometrical nonlinearity was shown to be the limiting mechanical nonlinear effect in the bridge resonators. In comparison, the BAW resonators demonstrated operation near the fundamental material limit for silicon. The BAW resonators were theoretically and experimentally shown to have three orders-of-magnitude larger energy storage capability than the analyzed flexural resonators. Moreover, the comparison to macro quartz crystals showed that for the silicon resonators the maximum energy density attainable is orders-of-magnitude larger. The importance of the energy storage capacity was demonstrated by estimating the theoretically attainable oscillator signal-to-noise ratio. The flexural resonators were shown to have inferior phase noise floor in comparison to macroscopic quartz crystals while the BAW resonators can rival the quartz crystal performance.

\section{ACKNOWLEDGMENT}

The authors would like to thank A. Alastalo for useful discussions and J. Kiihamäki for device processing.

\section{REFERENCES}

[1] C. T.-C. Nguyen, "Frequency-selective MEMS for miniaturized lowpower communication devices," IEEE Trans. Microwave Theory Techn. vol. 47, pp. 1486-1503, Aug. 1999.

[2] T. Mattila, J. Kiihamäki, T. Lamminmäki, O. Jaakkola, P. Rantakari, A. Oja, H. Seppä, H. Kattelus, and I. Tittonen, "12 MHz micromechanical bulk acoustic mode oscillator,” Sens. Actuators, Phys. A, vol. 101, no. 1-2, pp. 1-9, Sept. 2002.

[3] L. D. Landau and E. M. Lifshitz, Mechanics, 3rd ed. Oxford, U.K.: Butterworth-Heinemann, 1999.

[4] A. H. Nayfeh, Nonlinear Oscillations. New York: Wiley, 1979.

[5] A. Oja, T. Mattila, H. Seppä, J. Kiihamäki, T. Lamminmäki, M. Koskenvuori, P. Rantakari, and I. Tittonen, "Nonlinear effects in bulk acoustic mode microresonators," in Proc. Eurosensors'02, The 16th European Conference on Solid-State Transducers, Prague, Czech Republic, Sept. 15-18, 2002, pp. 381-382.

[6] W. Weaver Jr., S. Timoshenko, and D. Young, Vibration Problems in Engineering, 5th ed. New York: Wiley, 1990.

[7] P. Malatkar, "Nonlinear Vibrations of Cantilever Beams and Plates," Dissertation, 2003.

[8] C. Wilson and P. Beck, "Fracture testing of bulk silicon microcantilever beams subjected to a side load," J. Microelectromech. Syst., vol. 5, pp. 142-150, Sept. 1996.

[9] T. Veijola and T. Mattila, "Modeling of nonlinear micromechanical resonators and their simulation with the harmonic-balance method," Int. J. RF Microwave Comput.-Aided Eng., vol. 11, no. 5, pp. 310-321, Sep. 2001.

[10] K. Y. Kim and W. Sachse, "Nonlinear elastic equation of state of solids subjected to uniaxial homogeneous loading," J. Mater. Sci., vol. 35, no. 13, pp. 3197-3205, July 2000.

[11] H. McSkimin and P. Andreatch Jr., "Measurement of third-order moduli of silicon and germanium," J. Appl. Phys., vol. 35, no. 11, pp. 3312-3319, Nov. 1964.

[12] E. Anastassakis, A. Cantarero, and M. Cardona, "Piezo-Raman measurements and anharmonic parameters in silicon and diamond," Phys. Rev. $B$, vol. 41, no. 11, pp. 7529-7535, Apr. 1990

[13] S. Maas, Nonlinear Microwave and RF Circuits, 2nd ed. Norwood, MA: Artech House, 2003.

[14] V. Kaajakari, T. Mattila, A. Oja, J. Kiihamäki, H. Kattelus, M. Koskenvuori, P. Rantakari, I. Tittonen, and H. Seppä, "Square-extensional mode single-crystal silicon micromechanical RF-resonator," in Proc. Transducers'03, The 12th International Conference on Solid-State Sensors and Actuators, Boston, MA, June 8-12, 2003, pp. 425-432.

[15] T. Lee, The Design of CMOS Radio-Frequency Integrated Circuits. Cambridge, U.K.: Cambridge University Press, 1998.

[16] W. Robins, Phase Noise in Signal Sources (Theory and Applications), ser. IEE Telecommunication Series 9. New York: Peregrinus, 1982.

[17] J. Nosek, "Drive level dependence of the resonant frequency in BAW quartz resonators and his modeling," IEEE Trans. Ultrason., Ferroelect. Freq. Contr., vol. 46, pp. 823-829, July 1999.

[18] J. R. Vig and Y. Kim, "Noise in microelectromechanical system resonators," IEEE Trans. Ultrason., Ferroelect., Freq. Contr., vol. 46, pp. 1558-1565, Nov. 1999. 
[19] V. Kaajakari, T. Mattila, A. Oja, J. Kiihamäki, and H. Seppä, "Squareextensional mode single-crystal silicon micromechanical resonator for low phase noise oscillator applications," IEEE Electron Device Lett., vol. 25, no. 4, pp. 173-175, Apr. 2004.

[20] J. R. Vig and A. Ballato, "Frequency control devices," in Ultrasonic Instruments and Devices. New York: Academic, 1999.

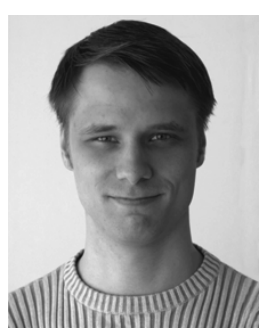

Ville Kaajakari received the M.S. and Ph.D. degrees in electrical and computer engineering from University of Wisconsin-Madison in 2001 and 2002, respectively.

$\mathrm{He}$ is currently Senior Research Scientist at VTT Information Technology, Finland, where his research interest is RF-MEMS.

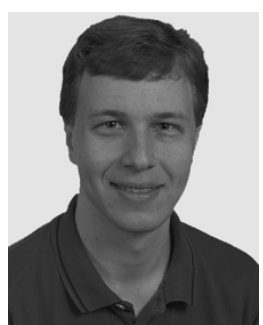

Tomi Mattila (M'00) received the M.Sc. and Dr.Tech. degrees from the Department of Technical Physics at Helsinki University of Technology, Finland, in 1994 and 1997, respectively.

Since 1999 he has been working as Senior Research Scientist at VTT Information Technology, Finland, where his current research interests concentrate on micromechanical RF-devices.

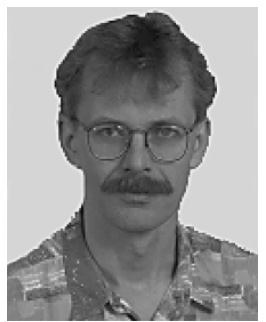

Aarne Oja received the Dr.Tech. degree in 1988 from the Low Temperature Laboratory of the Helsinki University of Technology, Finland.

Before joining VTT Information Technology, Finland, in 1995, he investigated nuclear magnetism at nanokelvin temperatures. He is the leader of MEMS Sensors group at VTT Microsensing and Research Professor since 2000. His current research interests include several microelectromechanical sensors and devices: RF-resonators, high-precision MEMS, ultrasound sensors, and magnetic MEMS.

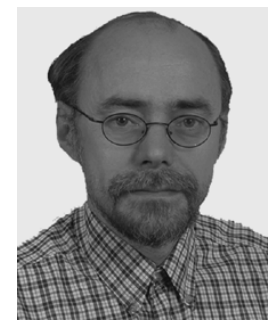

Heikki Seppä received the M.Sc., Lic.Tech., and Dr.Tech. degrees in technology, from Helsinki University of Technology, Finland, in 1977, 1979, and 1989, respectively.

He joined VTT Information Technology, Finland, in 1979 and since 1989, he has been employed there as a Research Professor. In 1994, he was appointed Head of the Measurement Technology field at VTT Automation. Since 2002, he has acted as Research Director, VTT Information Technology. He has done research work on electrical metrology, in general, and on superconducting devices for measurement applications, in particular. He is currently conducting research on dc-SQUID's, quantized Hall effect, SET-devices, RF-instruments, and microelectromechanical devices. 